\title{
Relationships between Comorbidity and Health-related Quality of Life Outcomes among Non-Hodgkin Lymphoma Survivors
}

Suyeon Lee, RN, MS푸 So Hee Kim, RN, MS²; Im-Ryung Kim, RN, OAPN, MSN³; Onam Ok, RN, MS4; Soo Hyun Kim, RN, PhD, OCN5

1 Department of Oncology, Asan Medical Center, Seoul, Korea ${ }^{2}$ Cancer Edu-info Center at Asan Medical Center, Seoul, Korea

${ }^{3}$ Cancer Education Center, Samsung Medical Center, Seoul, Korea ${ }^{4}$ Division of Hematology-Oncology, Samsung Medical Center, Seoul ,Korea ${ }^{5}$ Department of Nursing, Inha University, Incheon, Korea

\section{INTRODUCTION}

Many non-Hodgkin lymphoma (NHL) survivors are increasing due to earlier diagnosis and more effective treatment. NHL survivors have comorbidities that influence their quality of life. The aim of this study was to assess the prevalence of comorbidities and the association between comorbidities and the health-related quality of life (HRQoL) among Korean NHL survivors.

\section{METHODS}

A cross-sectional study was conducted among $570 \mathrm{NHL}$ survivors who had been treated at any of 3 Korean hospitals from 1989 through 2010. HRQoL was assessed with the European Organization for Research and Treatment of Cancer (EORTC) Quality of Life Questionnaire Core 30 (QLQ-C30). Comorbidity was assessed with Charlson Comorbidity Index.

\section{RESULTS}

\section{- Frequencies of comorbidity}

\begin{tabular}{lcc} 
Comodities & No. & $\%$ \\
\hline Number of comorbidities & & \\
0 & 223 & 39.1 \\
1 & 208 & 36.5 \\
2 & 86 & 15.1 \\
$\geq 3$ & 53 & 9.4 \\
Type of comorbidities & & \\
Cerebrovascular disease & 10 & 1.8 \\
Heart disease & 26 & 4.6 \\
Diabetes Mellitus & 70 & 12.3 \\
Liver disease & 47 & 8.2 \\
Lung disease & 19 & 3.3 \\
Hypertension & 113 & 19.8 \\
Gastrointestinal disease & 76 & 13.3 \\
Musculoskeletal disease & 84 & 14.7 \\
Renal disease & 24 & 4.2 \\
Others & 88 & 15.4 \\
Hyperlipidemia & 18 & 3.2 \\
Ocular disease & 12 & 2.1 \\
Peripheral neuropathy & 10 & 1.8 \\
Thyroid dysfunction & 8 & 1.4 \\
Prostate disease & 7 & 1.2 \\
\hline
\end{tabular}

- Adjusted mean scores for HADS, and BFI by number of comorbidities

\begin{tabular}{l|c|c|c|c} 
& \multicolumn{3}{|c|}{ Number of comorbidities } & \multirow{2}{*}{$p$} \\
\cline { 2 - 3 } & 0 & 1 & $\geq 2$ & \\
HADS & & & & \\
Anxiety & 3.82 & 4.95 & 5.48 & .000 \\
Depression & 4.19 & 5.40 & 5.90 & .000 \\
BFI & & & & \\
$\quad$ Total BFI score & 22.18 & 27.59 & 30.04 & .001 \\
\hline
\end{tabular}

Adjusted for age, sex, marital status, education status, employment status, monthly income, stage, time since diagnosis, previous treatment.
- Adjusted mean scores for EORTC QLQ-C30 by number of comorbidities

\begin{tabular}{lcc|c|c} 
& \multicolumn{3}{|c|}{ Number of comorbidities } & \multirow{2}{*}{$\mathbf{p}$} \\
\cline { 2 - 3 } & 0 & 1 & $\geq 2$ & \\
EORTC QLQ-C30 & & & & \\
\cline { 1 - 2 } Physical functioning & 86.72 & 84.88 & 80.19 & .001 \\
Role functioning & 87.49 & 84.55 & 80.44 & .021 \\
Emotional functioning & 84.72 & 77.61 & 76.70 & .000 \\
Cognitive functioning & 82.29 & 77.97 & 76.36 & .012 \\
Social functioning & 85.22 & 76.82 & 74.50 & .000 \\
GHS/QOL & 74.93 & 68.27 & 67.57 & .001 \\
Fatigue & 26.41 & 31.70 & 34.03 & .004 \\
Nausea and vomiting & 4.27 & 7.59 & 9.40 & .005 \\
Pain & 7.97 & 13.19 & 15.27 & .003 \\
Dyspnea & 10.85 & 14.29 & 16.82 & .052 \\
Insomnia & 14.91 & 18.41 & 25.56 & .004 \\
Appetite loss & 7.10 & 11.97 & 12.85 & .029 \\
Constipation & 14.94 & 15.67 & 19.30 & .308 \\
Diarrhea & 10.91 & 9.02 & 15.68 & .027 \\
Financial difficulties & 22.85 & 32.10 & 29.18 & .007 \\
\hline
\end{tabular}

Adjusted for age, sex, marital status, education status, employment status, monthly income stage, time since diagnosis, previous treatment.

Adjusted mean scores for EORTC QLQ-C30, HADS, and BFI by the presence of specific disease

\begin{tabular}{|c|c|c|c|c|c|c|c|c|c|}
\hline & \multicolumn{3}{|c|}{ HTN } & \multicolumn{3}{|c|}{ GI } & \multicolumn{3}{|c|}{ Muscular } \\
\hline & No & Yes & $p$ & No & Yes & p & No & Yes & $p$ \\
\hline \multicolumn{10}{|l|}{ EORTC QLQ-C30 } \\
\hline Physical functioning & 84.52 & 84.67 & .927 & 85.09 & 81.02 & .031 & 86.04 & 75.01 & .000 \\
\hline Role functioning & 84.35 & 86.71 & .333 & 85.63 & 79.31 & .020 & 86.17 & 75.98 & .000 \\
\hline Emotional functioning & 79.39 & 84.19 & .038 & 81.17 & 74.36 & .008 & 81.28 & 73.77 & .004 \\
\hline Cognitive functioning & 78.44 & 83.37 & .020 & 80.51 & 71.72 & .000 & 80.41 & 72.51 & .001 \\
\hline Social functioning & 79.37 & 81.12 & .557 & 81.01 & 70.95 & .003 & 80.75 & 72.67 & .017 \\
\hline GHS/QOL & 70.94 & 70.12 & .722 & 71.51 & 66.01 & .032 & 72.04 & 62.68 & .000 \\
\hline Fatigue & 31.26 & 24.97 & .009 & 28.98 & 37.39 & .002 & 28.96 & 37.40 & .002 \\
\hline Nausea and vomiting & 6.82 & 5.95 & .590 & 5.34 & 15.42 & .000 & 6.10 & 10.28 & .023 \\
\hline Pain & 11.60 & 11.35 & .913 & 10.61 & 17.92 & .004 & 10.11 & 20.79 & .000 \\
\hline Dyspnea & 13.56 & 13.14 & .863 & 13.15 & 15.64 & .362 & 12.83 & 17.61 & .083 \\
\hline Insomnia & 18.70 & 18.32 & .901 & 18.00 & 22.76 & .163 & 17.35 & 26.77 & .006 \\
\hline Appetite loss & 10.27 & 9.93 & .889 & 10.15 & 10.60 & .871 & 10.02 & 11.40 & .622 \\
\hline Constipation & 17.59 & 10.17 & .008 & 15.98 & 17.67 & .591 & 15.89 & 18.24 & .462 \\
\hline Diarrhea & 11.51 & 10.30 & .623 & 10.26 & 18.09 & .004 & 9.98 & 19.69 & .000 \\
\hline Financial difficulties & 27.78 & 27.48 & .932 & 26.36 & 36.62 & .007 & 26.92 & 32.94 & .124 \\
\hline \multicolumn{10}{|l|}{ HADS } \\
\hline Anxiety & 4.72 & 4.19 & .210 & 4.47 & 5.59 & .017 & 4.49 & 5.46 & .043 \\
\hline Depression & 5.02 & 5.06 & .933 & 4.86 & 6.12 & .007 & 4.88 & 5.96 & .023 \\
\hline \multicolumn{10}{|l|}{$\mathrm{BFI}$} \\
\hline Total BFI score & 26.58 & 23.30 & .120 & 25.01 & 32.34 & .002 & 24.65 & 34.53 & .000 \\
\hline \multicolumn{10}{|c|}{$\begin{array}{l}\text { Adjusted for age, sex, marital status, education status, employment status, monthly income, } \\
\text { stage, time since diagnosis, previous treatment. }\end{array}$} \\
\hline
\end{tabular}

Comorbidities are common health problems in NHL survivors. Comorbidities have significantly negative influence on QOL.

These findings suggested comprehensive care for NHL survivors. 